Relations industrielles

Industrial Relations

\title{
Voluntary Medical Care Insurance - A Study of non-profit plans in Canada. Ottawa, 1954, Research Division, Department of National Health and Welfare, 208 pp. Multilith.
}

\section{James Hodgson}

Volume 10, numéro 1, décembre 1954

URI : https://id.erudit.org/iderudit/1022795ar

DOI : https://doi.org/10.7202/1022795ar

Aller au sommaire du numéro

Éditeur(s)

Département des relations industrielles de l’Université Laval

ISSN

0034-379X (imprimé)

1703-8138 (numérique)

Découvrir la revue

Citer ce compte rendu

Hodgson, J. (1954). Compte rendu de [Voluntary Medical Care Insurance - A Study of non-profit plans in Canada. Ottawa, 1954, Research Division, Department of National Health and Welfare, 208 pp. Multilith.] Relations industrielles / Industrial Relations, 10(1), 64-64.

https://doi.org/10.7202/1022795ar

Tous droits réservés (C Département des relations industrielles de l’Université Laval, 1954
Ce document est protégé par la loi sur le droit d'auteur. L'utilisation des services d’Érudit (y compris la reproduction) est assujettie à sa politique d'utilisation que vous pouvez consulter en ligne.

https://apropos.erudit.org/fr/usagers/politique-dutilisation/ 
wherever tried, because no one wants to do the dirty work or exert himself strenuously. Communistic societies soon revert to the capitalistic principle, "From each according to his ability and industry and to each according to his ability and industry". Attractive as the doctrine of Communism fight be, no society has been able to distribute wealth according to its tenets without destroying individual initiative.

Un catholique aimerait évidemment voir sous la plume de Stokdyk des considérations qui dépassent le plan matériel du communisme et du libéralisme. Il est encore malheureux qu'il ait affirmé que le droit de propriété n'est pas un droit naturel mais le fruit d'une «sanction sociale». On regrette que, pris dans l'action comme il l'a été, il n'ait pas poussé davantage ses réflexions sur l'homme, l'homme tout entier, l'homme qui a su être lui-même en consacrant sa vie au service des autres. Il aurait réalisé à partir de sa propre expérience que, pour une élite au moins, lidéal de service est un stimulant capable de conduire à de fécondes réalisations.

\section{Charles Lemelin}

Voluntary Medical Care Insurance - A Study of non-profit plans in Canada. Ottawa, 1954, Research Division, Department of National Health and Welfare, 208 pp. Multilith.

On parlait à peine il y a quinze ans de l'assurance-santé. A part la Croix Bleue qui offrait une assurance-hospitalisation, à peu près rien ne se faisait au Canada dans ce genre d'assurance. Puis en 1937, nait le premier service d'assurance-maladie et d'autres de 1939 jusqu'à 1946, année où l'on voit l'éclosion de cinq des quatorze services actuellement en existence.

C'est l'histoire, l'organisation et le financement de ces services d'assurancemaladie qu'étudie cette publication due au Service de la recherche du Ministère de la santé nationale et du bien-être, qui a confié à John Osborne, économiste attaché au Service, le soin de préparer et rédiger ce travail.
Après un chapître consacré à l'historique de l'organisation de ces services, les débuts timides de l'avant-guerre, l'affermissement pendant la guerre et l'expansion formidable dans l'aprèsguerre, où l'on voit que le nombre de participants actifs et leurs dépendants passer de 13,000 en 1939 à plus de $2,000,000$ en 1953, l'auteur étudie longuement l'organisation de ces plans, qu'il divise en deux catégories: l'assurance service et l'assurance indemnité. Les sociétés d'assurance-service s'engagent comme dit l'auteur «to pay the full cost of medical care services rendered to their members. The latter plans usually enter into contracts with doctors (or their professional associtions) who agree to accept the fees paid by the plans as full payment for services rendered 》 (page 21) alors que les sociétés d'assurance indemnité «guarantee to reimburse the individual member for his medical care expenses up to a certain fixed maximum sums for each type of service received, and do not enter into contracts with doctors to accept the amounts paid by the plans as full payment for services rendered (ibidem).

Nous connaissons dans la province de Québec deux sociétés d'assurance-santé, Les Services de santé de Québec et la Quebec Hospital Service Association, établie comme service d'assurance-santé après plusieurs années de fonctionnement comme service d'assurances-hospitalisation. La première offre de l'assurance service et la seconde de l'assurance indemnité.

Les troisième et quatrième chapitres sont consacrés à l'étude du financement des sociétés et de l'utilisation des services offerts. Une étude complète, détaillée et précise de tous les aspects: revenus, dépenses, bénéfices, administration, paiements aux médecins, quantité et valeur des services utilisés.

L'étude soignée et bourrée de chiffres et de faits indigestes, se termine par une conclusion qui offre un bon résumé, une excellente vue d'ensemble de la situation.

Regrettons que la présentation des tableaux soit aussi peu soignée, ce qui les rend difficiles à consulter.

JAMES Hodgson 